SHORT REPORT

\title{
BMI distribution changes in adolescent British girls
}

\author{
E Georgiades, J J Reilly, E Stathopoulou, A M Livingston, Y P Pitsiladis
}

Arch Dis Child 2003;88:978-979

Body mass index (BMI) distribution changes were assessed in 2547 relatively affluent English girls, aged 12-16 years, during the UK childhood obesity epidemic (1986-96). An increase in BMI variability was observed only in 12-14 year olds, suggesting that $\mathrm{BMl}$ changes for population subgroups were complex, and inconsistent with a generalised increase in BMI.

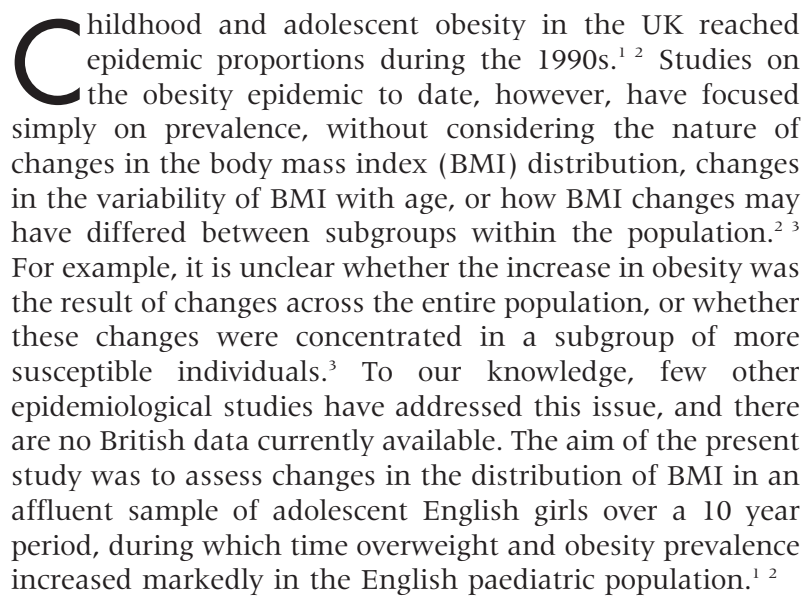

\section{METHODS}

Anthropometric data were collected from 2547 pupils, aged 12-16 years, from a girls secondary school in a relatively affluent area of Kent, over a 10 year period (1986-96). The study was approved by the ethics committee of the West Kent Health Authority. Measurements were conducted by physical education (PE) teachers, once every term throughout attendance at the school, with the girls dressed in light PE clothing. Weight and height were measured (to the nearest $0.1 \mathrm{~kg}$ and $0.1 \mathrm{~cm}$, respectively) using Seca weighing scales and a wall mounted stadiometer (Seca-Weighing and Measuring Systems, Birmingham, UK). The first and the last three years of data collected were combined, forming two groups for comparison in the present study: collection years, 1986-88 and 1994-96.

Following a test of normality, BMI data were transformed by taking the natural logs of both groups; two-sample $t$ tests were used to assess differences between the groups. The BMI data are presented as the antilog of the mean (geometric means) \pm 1 standard deviation (SD). Mean difference plots were used to assess BMI distributions. A mean difference plot is a graphical method for comparing two distributions; it shows the direction and magnitude of any shift between distributions. The points on the plot represent the difference between the two distributions (1994-96) - (1986-88) at a given centile level, plotted against the means of the same centile level from the two distributions. ${ }^{3}$ Changes in these plots over time can indicate differences in the variability of BMI over time, or in skewness of the BMI distribution over time. The BMI data from the two groups were also converted to SD scores using the national reference data (the British 1990 growth reference), allowing for comparison of the whole dataset, adjusted for age, between the two collection periods.

\section{RESULTS}

There were no significant differences in mean BMI values between the two groups, for all ages (table 1), although values for mean BMI measured during 1994-96, were consistently lower than corresponding 1986-88 data. When comparing the converted BMI values to SD scores using the national reference data, however, a small but statistically significant difference was found between the two groups. The 1994-96 values (mean 0.16 (SD 1.14)) were significantly lower than the corresponding 1984-86 values $(0.25(0.96))(\mathrm{p}=0.032)$.

Figure 1 shows mean difference plots, comparing the BMI distribution for 1986-88 data and 1994-96 data, for each age group separately. Although there is some evidence of increased skewness in plots for 13 and 14 year olds, the amount of curvature is small and probably non-significant; a much larger sample size would be required to confirm this. All plots, therefore, appear to be essentially linear, this trend indicating an increase in BMI variability between the two distributions, which is more apparent in 12-14 year olds. The 1994-96 SDs for 12-14 year olds (table 1) also appear to be increased compared to 1986-98, confirming the trends observed in the mean difference plots. This apparent increase in SDs and, hence, BMI variability, however, seems to be transient, as similar differences were not observed in the 1516 year olds (table l; fig 1 ).

\section{DISCUSSION}

The present study found no evidence of an increase in average BMI across the 10 year period of data collection, which incidentally coincided with an epidemic of childhood and adolescent obesity in the UK. ${ }^{12}$ Research on the epidemic has tended to focus on the proportion of a given sample above fixed BMI cut offs, such as the 95th centile, not on changes in mean or median BMI, or on the distribution of BMI. $^{23}$ Within the present samples of adolescent girls studied, the absence of any increase in average BMI is

Table 1 BMI for girls aged 12-16 years, in 1986-88 and 1994-96

\begin{tabular}{llll}
\hline Age & $1986-88$ & $1994-96$ & $p$ value \\
\hline 12 & $18.9(2.4)$ & $18.5(3.1)$ & $\mathrm{p}=0.25$ \\
& $(\mathrm{n}=252)$ & $\begin{array}{l}(\mathrm{n}=184) \\
19.7(3.3)\end{array}$ & $\mathrm{p}=0.88$ \\
13 & $19.7(2.8)$ & $(\mathrm{n}=330)$ & \\
& $(\mathrm{n}=248)$ & $20.2(3.4)$ & $\mathrm{p}=0.62$ \\
14 & $20.3(2.7)$ & $(\mathrm{n}=337)$ & \\
& $(\mathrm{n}=253)$ & $20.5(2.7)$ & $\mathrm{p}=0.29$ \\
15 & $20.8(2.7)$ & $(\mathrm{n}=275)$ & \\
& $(\mathrm{n}=269)$ & $20.9(2.5)$ & $\mathrm{p}=0.58$ \\
16 & $21.1(2.7)$ & $(\mathrm{n}=155)$ & \\
\hline
\end{tabular}

Values quoted are geometric means ( \pm 1 SD) (after back transformation). 

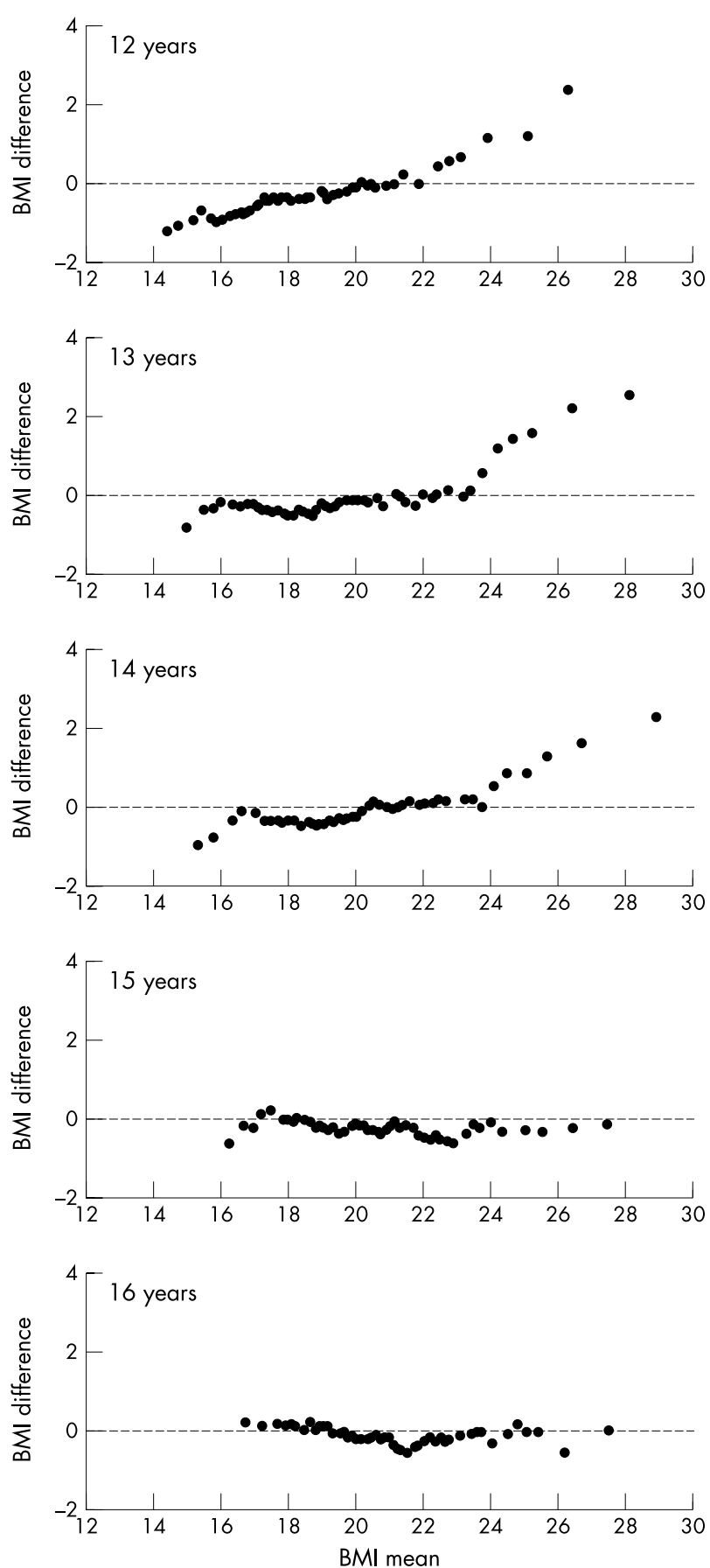

Figure 1 BMI distribution mean difference plots for girls aged 12-16 years.

unexpected. Indeed, the results of the comparison of the converted BMI values to SD scores using the national reference data (adjusted for age), indicate a smaller deviation from the British reference values in the 1994-96 dataset compared to the 1984-86 values, although both datasets do not appear appreciably different from the national reference data. The population studied may have been relatively unaffected by the epidemic, or the epidemic may have been delayed in more affluent socioeconomic groups. Alternatively, the absence of significant change in the mean (with a trend to lower mean BMI over time) may be consequential to the fact that BMI is a fairly crude measure, which reflects both fat and fat free mass. The results, therefore, could simply indicate a reduction in fat free mass in the population studied, over the period of data collection.

Two other recent studies of British children have suggested that lean body mass accretion has declined over time. ${ }^{45}$ In our study, absence of change in BMI may have masked real changes in body composition over the study period. ${ }^{4}$ This illustrates the potential value of collecting data on body composition, rather than just BMI in surveys. ${ }^{45} \mathrm{~A}$ similar pattern of negligible change or small decline in median BMI was reported for representative samples of British children in 1974-94. ${ }^{2}$ Regardless of the underlying explanation, the absence of change in average BMI in the present study indicates a more complex picture of population changes in nutritional status than that suggested by studies which have simply reported on changes in obesity prevalence.

The mean difference plots (fig l) showed that, in our sample, a transient increase in variability of BMI occurred in the 12-14 year olds at the end of the study period, but that this pattern was not present in the 15-16 year olds. The implications of this observation are unclear. Similar patterns have been observed in some populations, but not others, in an international review of BMI distribution differences between populations. ${ }^{6}$ A recent study suggested that the BMI distribution of children in the USA appears to have become more skewed over the course of the obesity epidemic. ${ }^{3}$ However, it is likely that the trends observed by the authors simply reflect increased variability in BMI, as in the present study, rather than increased "skewness" per se.

Larger studies on additional samples are therefore required to confirm the evidence presented here on BMI distribution changes, and to test for differences between subgroups within the population. An improved understanding of the nature of population changes in BMI distribution and, more precisely, changes in population body composition, may provide valuable guidance on obesity prevention, help identify groups at high or low risk of childhood obesity, and elucidate the precise causes of the obesity epidemic.

\section{ACKNOWLEDGEMENTS}

The authors are extremely grateful to Professor Tim Cole (Institute of Child Health, London) for his invaluable contribution to the analysis and data interpretation. We thank Fumi Varyani and Jennifer Mitchell for their initial help with the data entry. Adam Livingston was a recipient of a Wellcome Trust-Vacation Scholarship during the completion of this work.

\section{Authors' affiliations}

E Georgiades, A M Livingston, Y P Pitsiladis, University of Glasgow, Centre for Exercise Science and Medicine, Institute of Biomedical and Life Sciences, Glasgow G12 8QQ, UK

J J Reilly, University of Glasgow, Dept of Human Nutrition, Yorkhill Hospitals, Glasgow G3 8SJ, UK

E Stathopoulou, Medway Maritime Hospital, Community Child Health, Gillingham, Kent ME7 5NY, UK

\section{Correspondence to: Dr Y P Pitsiladis; Y.Pitsiladis@bio.gla.ac.uk}

Accepted 13 January 2003

\section{REFERENCES}

1 Reilly JJ, Dorosty AR. Epidemic of obesity in UK children. Lancet 1999;354:1874-5.

2 Chinn S, Rona RJ. Prevalence and trends in overweight and obesity in three cross-sectional studies of British children, 1974-1994. BMJ 2001;322:24-6.

3 Flegal KM, Troiano RP. Changes in the distribution of body mass index of adults and children in the US population. Int J Obes 2000;24:807-18.

4 Wells JCK, Coward WA, Cole TJ, et al. The contribution of fat and fat free tissue to body mass index in contemporary children and the reference child. Int J Obes 2002;26:1323-8.

5 Ruxton CH, Reilly JJ, Kirk TR. Body composition of healthy 7-8 year old children and a comparison with the "reference child". Int J Obes 1999;23:1276-81.

6 Cole TJ, Bellizzi MC, Flegal KM, et al. Establishing a standard definition for child overweight and obesity: international survey. BMJ 2000;320:1240-3. 